\title{
Quality Control of Natural Products by Fingerprinting of Eastern Blotting
}

Shunsuke Fujii ${ }^{1}$, Nguyen Huu Tung ${ }^{1}$, Takuhiro Uto ${ }^{1}$, Hiroyuki Tanaka ${ }^{2}$, Xiao-Wei $\mathrm{Li}^{3}$, Shao-Qing Cai ${ }^{3}$, Waraporn Putalum ${ }^{4}$ and Yukihiro Shoyama $^{1 *}$

${ }^{1}$ Faculty of Pharmaceutical Sciences, Nagasaki International University, 2825-7 Huis Ten Bosch, Sasebo, Nagasaki, 859-3298, Japan

${ }^{2}$ Department of Pharmacognosy, Graduate School of Pharmaceutical Sciences, Kyushu University, 3-1-1 Maidashi, Higashi-ku, Fukuoka, 812-8582, Japan

${ }^{3}$ State Key Laboratory of Natural and Biomimetic Drugs, Peking University, Beijing 100191, P.R. China

${ }^{4}$ Research Group for Pharmaceutical Activities of Natural Products using Pharmaceutical Biotechnology (PANPB), Faculty of Pharmaceutical Sciences, Khon Kaen University, Khon Kaen, Thailand

"Corresponding author: Yukihiro Shoyama, Faculty of Pharmaceutical Sciences, Nagasaki International University, 2825-7 Huis Ten Bosch, Sasebo, Nagasaki, 859-3298, Japan, Tel: +81956205622; Fax: +8156205740; E-mail: shoyama@niu.ac.jp

Received date: June 28, 2016; Accepted date: Jul 26, 2016; Published date: Jul 28, 2016

Copyright: (C) 2016 Yukihiro S, et al. This is an open-access article distributed under the terms of the Creative Commons Attribution License, which permits unrestricted use, distribution, and reproduction in any medium, provided the original author and source are credited.

\begin{abstract}
Eastern blotting was developed for small molecule compounds which have no affinity of conjugation to membrane instead of western blotting. Crude extracts of medicinal plants were developed by TLC plate followed to be blotted to polyvinylidene fluoride or polyether sulfone membrane by heating. Components on membrane were conjugated with carrier protein resulting in fixing to membrane. Staining was followed to western blotting system using monoclonal antibody (MAb). Four types of natural products, solasodine glycosides, aristrochic acid, glycyrrhizin-liquiritin and ginsenosides were discussed in this review. 1) Solasodine glycosides are selected as an example of staining for almost all solasodine glycosides using the anti-solamargine MAb having wide cross-reactivity. 2) Aristrochic acids possess no capacity of conjugation with membrane and their aqueous solubility is quite poor. To improving them, aristrochic acids should be synthesized for conjugation with carrier protein in order to combine to membrane. 3) Two different types of marker components in licorice, triterpene glycoside; glycyrrhizin and flavonoid glycoside; liquiritin can be detected by double eastern blotting. 4) Ginsenosides are also detected by double eastern blotting and this system might be possible to clear the distribution of ginsenosides in tissue for understanding of pharmacological activity.
\end{abstract}

Keywords: Eastern blotting; Monoclonal antibody; Solasodine glycoside; Aristolochic acid; Glycyrrhizin; Liquiritin; Ginsenoside

\section{Introduction}

Recently immunoassay systems using monoclonal antibody (MAb) against drugs and bioactive compounds having small molecular weight have become an important tool in wide field. We prepared many MAbs against natural products like forskolin [1], solamargine [2], crocin [3], marihuana compound [4], opium alkaloids [5], ginsenosides [6,7], berberine [8], sennosides $[9,10]$, paeoniflorin $[11,12]$, glycyrrhizin (GC) [13,14], saikosaponin [15-17], ginkgolic acid [18], aconitine alkaloid [19] and baicalin [20]. More recently MAbs against capsaicinoids [21], artemisinin [22,23], luteoloside [24], daidzin [25] and puerarin [26] have been prepared and developed individual competitive enzyme-linked immunosorbent assay (ELISA) as a high sensitive, specific, and simple methodology resulting in screening of medicinal plants targeting bioactive component [27], pharmacokinetics for capsaicinoids [21] artemisinin [28], and ginsenoside Rb1 and $\operatorname{Rg} 1$ [29].

The one-step purification of the target component which is an antigen molecule like forskolin [30], ginsenoside Rb1 [31], solasodine glycosides [32], GC [33] and naringin [34] by immunoaffinity column conjugated with MAb has been succeeded. In the case of GC the extract eliminated GC was tested the biological activity and confirmed that the extract seemed to be a knockout extract [35-37].

As an immunostaining technique to detect higher molecular compounds like peptides and proteins using MAb western blotting is used widely. However, since small molecular compounds are ruled out from western blotting, a new staining system for small molecule compounds might be required. Therefore we separated the function of western blotting into 3 steps; separation of compounds by thin-layer chromatography (TLC), blotting to polyvinylidene fluoride (PVDF) or polyether sulfone (PES) membrane and finally staining by MAb. Since blotted compounds on PVDF or PES membrane are easily washed out by washing, the compounds on membrane were combined with carrier protein resulting in fixing on membrane and then staining. For the conjugation of antigen molecule to carrier protein two methods were used; one is oxidative cleavage of sugar moiety releasing the aldehyde group which can be conjugated to carrier protein, and the other is expanding of function by appropriate linker resulting in an antigen molecule and carrier protein conjugate on the membrane. This methodology has been applied for several natural products like solasodine glycosides in Solanum spp. [38], saikosaponin in Bupleurum spp. [39], sennosides in Rheum spp. [40], ginsenosides in Panax spp. [41,42], GC [14] and liquiritin (Liq) [43] and so on. Therefore, we will discuss four types of natural products, solasodine glycosides, aristrochic acid, GC and ginsenosides in this review. Solasodine glycosides are selected as an example of staining for almost all solasodine glycosides using the MAb having wide cross-reactivity. Aristrochic acid should be synthesized for conjugation with carrier protein in order to combine to membrane compared to the other groups. In the case of licorice we selected two different types of marker components, triterpene glycoside; GC and flavonoid glycoside; Liq for fingerprinting by double eastern blotting. Final ginsenosides are the delegation of double eastern blotting for recognizing of pharmacological activity. 
Citation: Shunsuke Fujii, Nguyen Huu Tung, Takuhiro Uto, Hiroyuki Tanaka, Xiao-Wei Li, et al. (2016) Quality Control of Natural Products by Fingerprinting of Eastern Blotting. Pharm Anal Acta 7: 494. doi:10.4172/2153-2435.1000494

Page 2 of 11

\section{Solanum species}

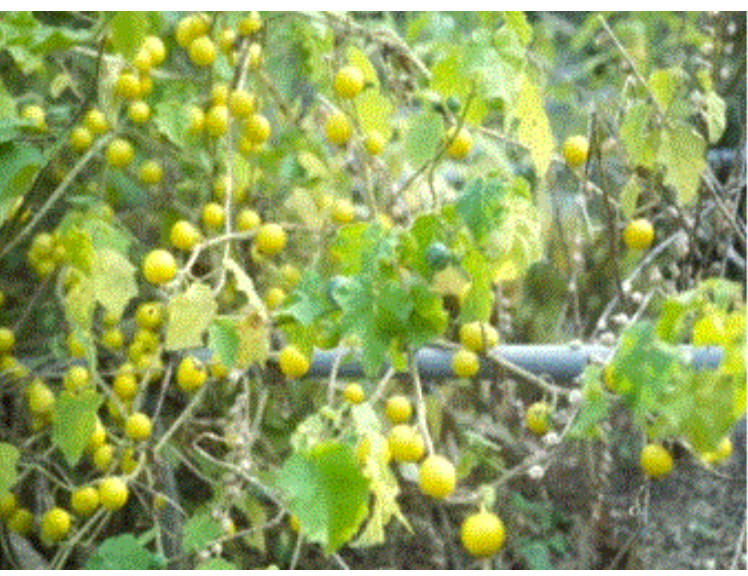

Figure 1: Solanum khasianum C. B. Cl. and fruiting.

The natural resources of adrenocortical and sex hormones, which are mainly obtained from diosgenin purified from Dioscorea saponin, are decreasing. The most important feature of solasodine is that it can be converted to dehydropregnenolone. Solasodine is found with a series of sugar residues attached to the oxygen at the C-3 position. The most common forms are the triglycosides, solamargine being predominant [44]. Therefore, the steroidal alkaloid glycosides of the solasodine type like solamargine have become important as a starting material for the production of steroidal hormones in the market. In order to analyze the concentration of solasodine glycosides in Solanum spp. (Figure 1). we prepared MAb against solamargine having wide cross reactivity for almost all solasodine glycosides [2] which can be used as resources for steroidal hormones. Figure 2 indicates the crossreactivities of MAb against solamargine. Other steroidal compounds, however did not cross-react with anti-solamargine MAb. Judging from above findings, it is clear that an aglycone and part of the glucose are immunogenetic resulted in that almost all solasodine type glycosides can be detected by anti-solamargine MAb.

Solasodine glycosides were applied to two TLC plate and developed by developing solvent system [38]. One plate was sprayed and colored with $\mathrm{H}_{2} \mathrm{SO}_{4}$. The other plate was blotted to a PVDF membrane by heating. The PVDF membrane was treated with sodium periodate releasing aldehyde group in a molecule followed to add BSA solution. Conjugate reaction occurred between aldehyde and BSA to give a shif base which combines to PVDF membrane. Figure 3 shows the pathway of solasodine glycoside-BSA conjugate on PVDF membrane. 
Citation: Shunsuke Fujii, Nguyen Huu Tung, Takuhiro Uto, Hiroyuki Tanaka, Xiao-Wei Li, et al. (2016) Quality Control of Natural Products by Fingerprinting of Eastern Blotting. Pharm Anal Acta 7: 494. doi:10.4172/2153-2435.1000494
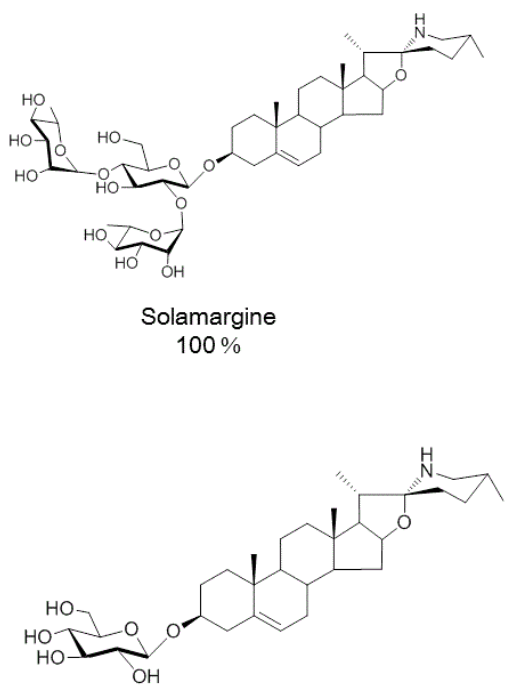

3-O- $\beta$-D-Glucopyranosyl solasodine $113 \%$

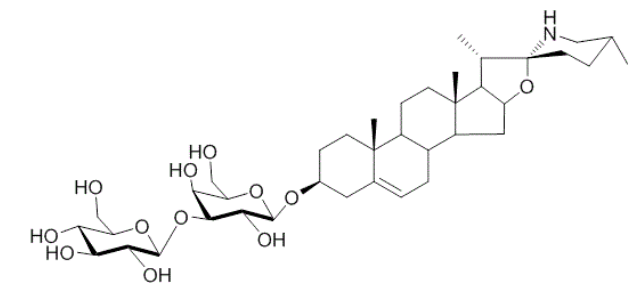

O- $\beta$-D-Glucopyranosyl-(1-3)-3-O- $\beta$-D-galactopyranosyl solasodine $118 \%$

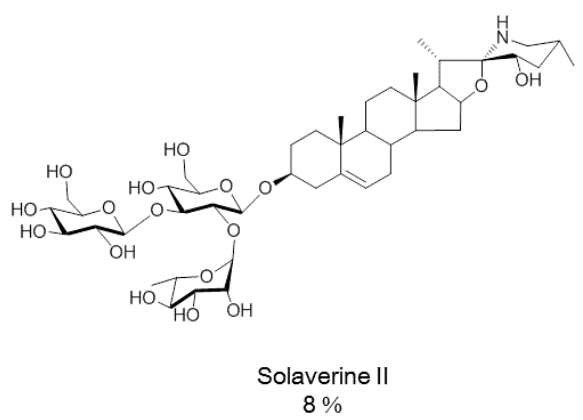

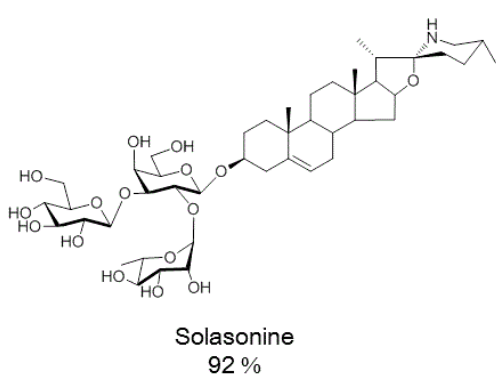
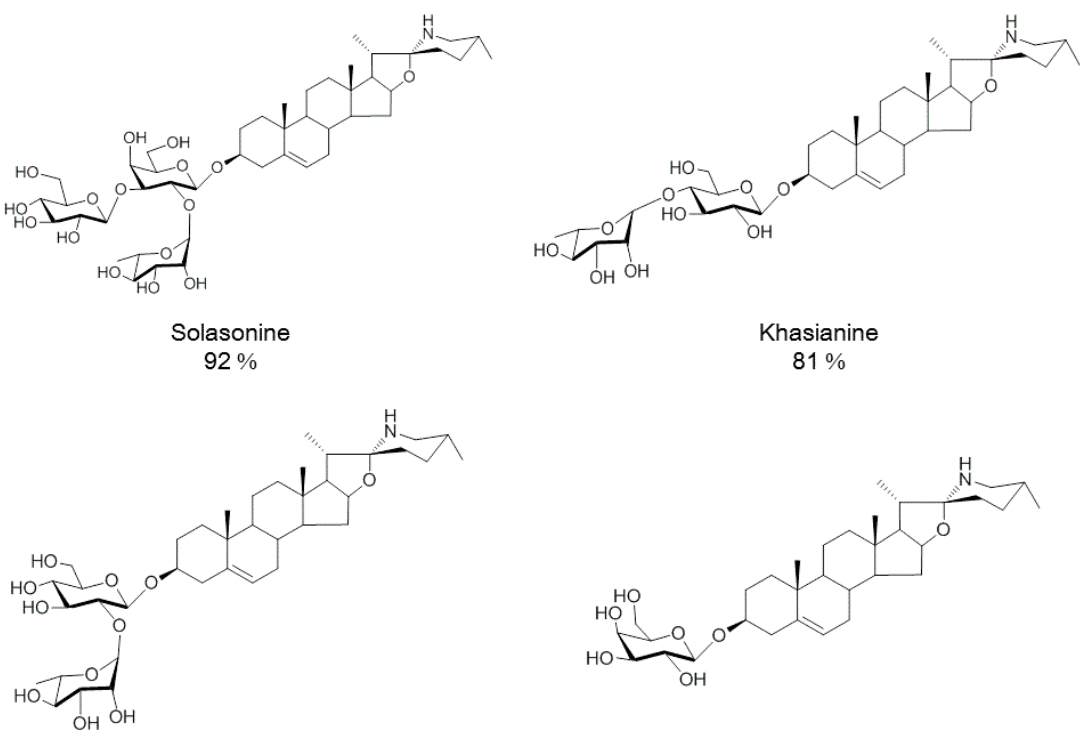

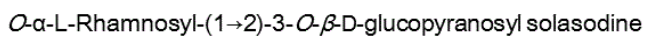

$98 \%$

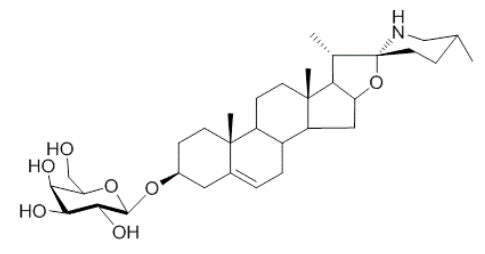

3-O- $\beta$-D-Galactopyranosyl solasodine $115 \%$

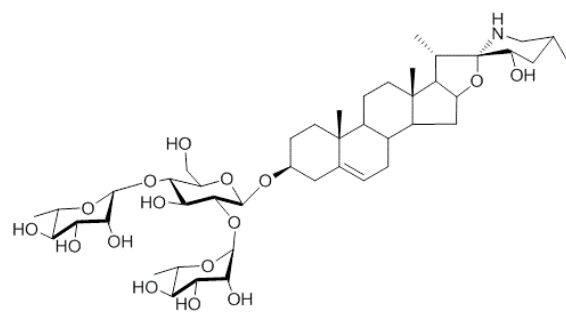

Solaverine I

olasodine

$44 \%$

Figure 2: Cross-reactivity of MAb against steroidal alkaloids. 


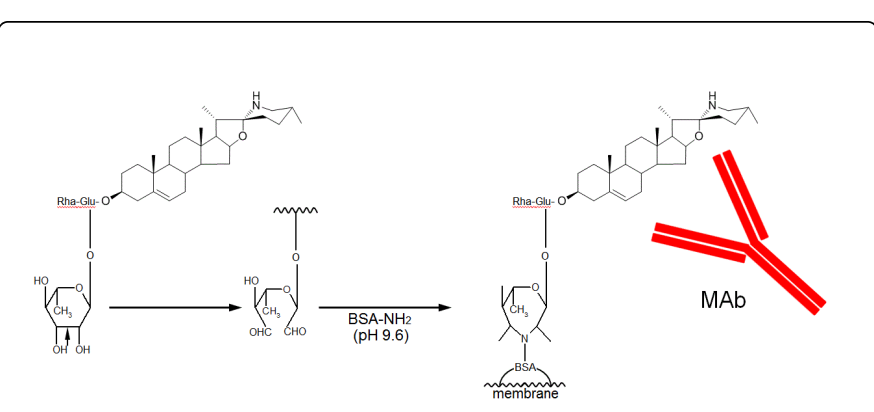

Figure 3: Schematic pathway of solasodine glycoside for eastern blotting.

In order to confirm the preparation of solamargine-BSA conjugate on the PVDF membrane, the band corresponding to the solamargineBSA conjugate was assessed by MALDI mass spectrometry. A broad peak of solamargine-BSA conjugate appeared at around m/z 69043 in MALDI mass spectrometry indicating clear conjugation between solamargine and BSA occurred because BSA molecule is 66464 . Therefore, it becomes clearly that the sugar moiety which was conjugated with BSA is necessary in this staining system. Then after anti-solamargine MAb was added to the membrane which was treated with peroxidase labeled secondary $\mathrm{MAb}$ and a substrate. Figure 4 indicated the fingerprinting by eastern blotting for several solasodine glycosides using anti-solamargine MAb. All of solasodine glycosides can be stained.
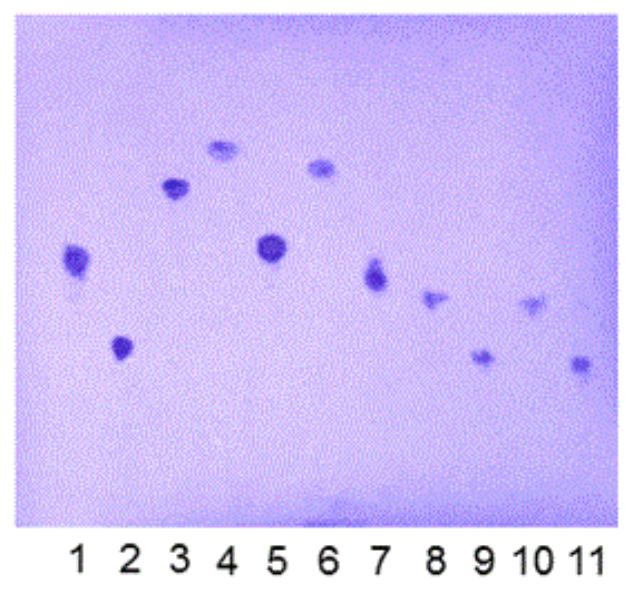

Figure 4: Fingerprinting by eastern blotting for several solasodine glycosides using anti-solamargine MAb. Lines 1 to 11 are solamargine, solasonine, khasianine, 3-O- $\beta$-D-glucopyranosyl solasodine, $\quad \mathrm{O}-\alpha$-L-rhamnosyl- $(1 \rightarrow 2)-3-\mathrm{O}-\beta$-D-glucopyranosylsolasodine, $\quad 3-\mathrm{O}-\beta-\mathrm{D}$-galacopyranosyl-solasodine, $\quad \mathrm{O}-\beta-\mathrm{D}-$ glucopyranosyl-( $1 \rightarrow 3)-3-\mathrm{O}-\beta-\mathrm{D}$-galacopyranosyl-solasodine,

solaverine I, solaverine II, 12-hydroxysolamargine and 12hydroxysolasonine, respectively. Two $\mu \mathrm{g}$ of samples were applied for TLC, respectively.

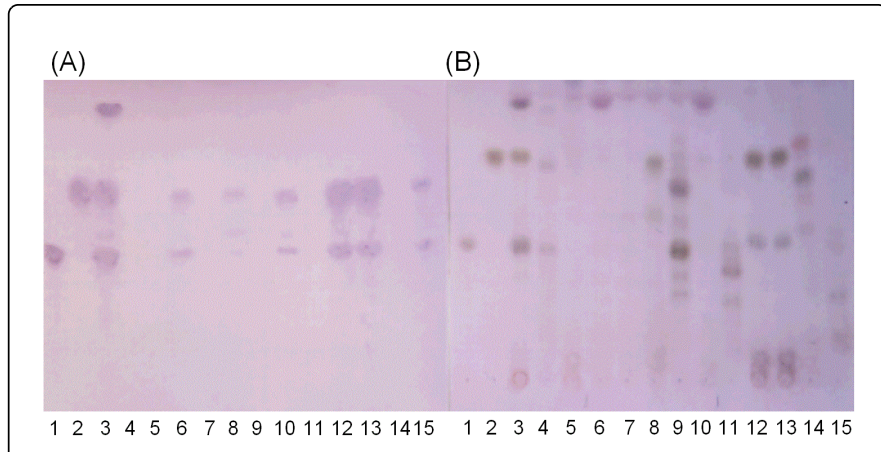

Figure 5: The eastern blotting (A) and TLC stained with $\mathrm{H}_{2} \mathrm{SO}_{4}$ (B) of Solanum spp. Lines 1 to 15 are solasonine, solamargine, $S$. khasianum, S. lyratum, S. spirale, S. melongena, S. stramonifolium, $S$. verbascifolium, $S$. indicum, $S$. aculeatissimum, $S$. trilobatum, $P$. minima, $S$. wrightii, $S$. torvum and $S$. nigrum, respectively.

Figure 5A showed the fingerprinting of Solanum spp. by eastern blotting using anti-solamargine MAb. It clearly showed that solamargine and solasonine are major solasodine glycosides in Solanum spp. and solasodine mono-glycoside can be detected in only S. khasianum. On the other hand the fingerprinting by $\mathrm{H}_{2} \mathrm{SO}_{4}$ (Figure 5B) indicated complicated pattern, because that $\mathrm{H}_{2} \mathrm{SO}_{4}$ detected the other type of glycosides like triterpene and/or steroidal components. The present methods are useful to determine solasodine-type glycoalkaloids. The combination assay method of their ELISA [2] and eastern blotting can be routinely used to survey natural resources for solasodine glycosides as a simple and rapid analysis. As we previously reported when analysis of lower concentrations of solasodine glycosides is required an immunoaffinity column conjugated with antisolamargine $\mathrm{MAb}$ is available. The elution from the column concentrates the solasodine glycosides. Therefore, an immunoaffinity column, ELISA and eastern blotting represent strong methodology for detection, quantitative analysis, and single step isolation for total solasodine glycosides [33].

\section{Aristolochia species}

Aristolochic acids (AAs), naturally occurring nephrotoxicants and human carcinogens, are associated with the development of a rapidly progressive interstitial nephritis called aristolochic acid nephropathy (AAN) [45], and may also be a cause for the Balkan Endemic Nephropathy (BEN) [46](Figure 6). AAs are a mixture of structurally related nitrophenanthrene carboxylic acids [47]. Among them, aristolochic acid I (AA-I, Figure 7) and its 8-demethoxylated form, aristolochic acid II (AA-II, Figure 7), are considered to be of particular significance $[48,49]$. 


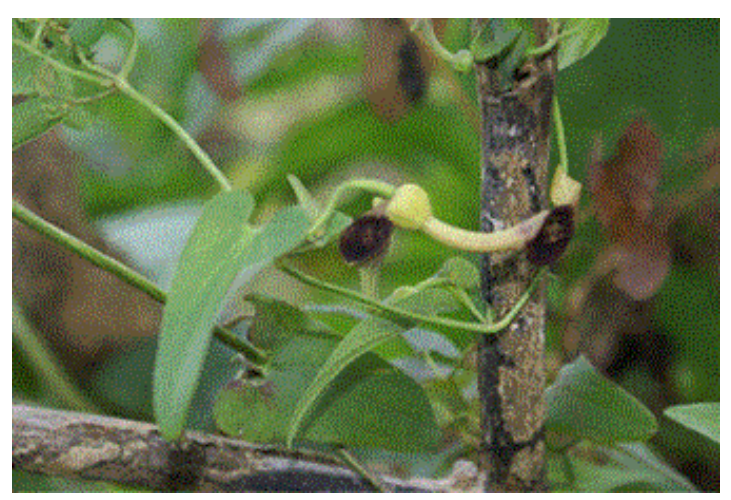

Figure 6: Aristolochia debilis Sieb. et Zucc. and its flowering.<smiles>[R]c1cc2c(cc([R5])c3c([R])cc4c(c32)OCO4)c([R])c1[R]</smiles>

AA-I $\mathrm{R}_{1}=\mathrm{COOH}, \mathrm{R}_{2}=\mathrm{H}, \mathrm{R}_{3}=\mathrm{H}, \mathrm{R}_{4}=\mathrm{OCH}_{3}, \mathrm{R}_{5}=\mathrm{NO}_{2}$ AA-II R1 $=\mathrm{COOH}, \mathrm{R}_{2}=\mathrm{H}, \mathrm{R}_{3}=\mathrm{H}, \mathrm{R}_{4}=\mathrm{H}, \mathrm{R}_{5}=\mathrm{NO}_{2}$

Figure 7: Structures of aristolochic acids.

It has been revealed that both AA-I and AA-II possess nephrotoxicity and/or genotoxicity [50-52]. AA-I and AA-II were found in the herbal medicines of Aristolochiaceae plants including Aristolochia and Asarum species [45,47,53], which have been prescribed for the traditional Chinese medicines (TCM) for centuries. Since the first evidence that the interstitial renal fibrosis rapidly progressed to end-stage of renal disease caused by the misuse of a slimming regimen containing AAs was reported in Belgium during 1990s [54], many countries started to regulate the herbs and supplements derived from Aristolochia and Asarum species. Although several species are listed in Chinese Pharmacopoeia in China, the number has been decreasing. Asiasarum sieboldii F. Maekawa and Asiasarum heterotropoides F. Maekawa var. mandshuricum F. Maekawa are still listed in the Japanese Pharmacopoeia (2011 edition) and severe quality control is described such as no detection of AAs by HPLC. In order to minimize the potential health risk aroused by AAs, a simple and sensitive method allowing visual detection might be highly required for the screening of a large number of herbal samples containing AAs. Detection methodology of AA-I and AA-II has been extensively reported, mainly focusing on the development of HPLC methods [55-57]. Compared to HPLC analysis, immunoassay systems have been proving simpler, more environmental-friendly and more rapid. For those benefits, ELISA using anti-AAs polyclonal antibody (PAb) [58] and anti-AA-I and AA-IVa MAb [59] have been reported previously. We also prepared anti-AA-II MAb [60] and anti-AA-I and -
II MAbs [61], and then investigated the localization of aristolochic acids in mice kidney [62] resulting in finding of $a$-actinin-4 as a possible target protein for aristolochic acid I in human kidney cell [63]. However, no immunostaining technique utilizing anti-AA MAb or anti-AA PAb has succeeded yet, due to the fact that AAs possess no capacity of conjugation with membrane and their aqueous solubility is quite poor. AAs, as the small molecules, will be easily washed out by buffer solutions during immunostaining process without fixation as previously described in solasodine glycosides which can be cleavage on sugar moiety to give aldehyde group in a molecule. Therefore, special fixing step was necessary so that AA could be blotted onto a membrane from the developed TLC plate. To facilitate the fixation to membrane, a modified carboxyl activation method was employed. 1-Ethyl-3-(3'dimethylaminopropyl) carbodiimide hydrochoride (EDC) method was commonly used to link the carboxyl groups of small molecular compounds to the amine groups of protein. We have been successfully prepared for immunization for various kinds of conjugates, succinate method like forskolin [64] and crocin [3], and conjugate with BSA through alanine in marihuana compound [65]. Considering the fact that the addition of $\mathrm{N}$-hydroxysuccinimide (NHS) could improve the stability of the intermediate and enhance the yield [66], the routine procedure was thereby improved by the addition of NHS together with EDC. After adding NHS, the carboxyl group of AA-I could be activated to form a semi-stable NHS ester. This intermediate can be coupled with the amine groups of carrier protein like BSA, resulting in the formation of AA-I-BSA conjugate which can be fixed on the PES membrane.

Figure 8 indicated the pathway of AA-1-BSA conjugate preparation which created a function of conjugating with PES membrane. The membrane was stained by the same way as previous solasodine glycosides using anti-AA-I MAb, secondary MAb and finally substrate, 4-chloro-1-naphthol. The other TLC plate developed was detected by UV light at $254 \mathrm{~nm}$.

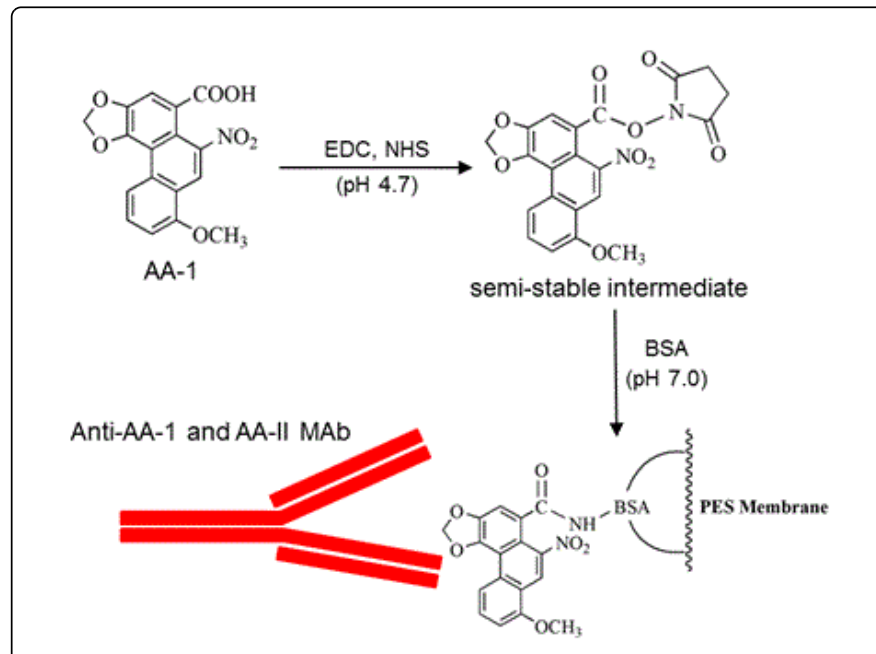

Figure 8: Scheme of synthetic pathway of AA-I and AA-II conjugate with BSA.

Figure 9 indicated the fingerprinting profiles of plant extracts by eastern blotting and TLC plate detected by UV light at $254 \mathrm{~nm}$. Although many spots including AAs and ALs were detected in Aristolochia species at $254 \mathrm{~nm}$ (Figure 9B), only AA-I and AA-II were detected by eastern blotting (Figure 9A). As shown in Figure 9A the 
Citation: Shunsuke Fujii, Nguyen Huu Tung, Takuhiro Uto, Hiroyuki Tanaka, Xiao-Wei Li, et al. (2016) Quality Control of Natural Products by Fingerprinting of Eastern Blotting. Pharm Anal Acta 7: 494. doi:10.4172/2153-2435.1000494

Page 6 of 11

stem of $A$. manshuriensis (Lane 1), the root of $A$. cinnabarina (Lane 3 ) and the root of $A$. contorta (Lane 4) were stained clearly on the membrane, whereas the root of $A$. fangchi (Lane 2), the root of $A$. debilis (Lane 5), the whole plant of $A$. mollissima (Lane 6) and the fruit of $A$. contorta (Lane 8 ) were stained weakly. On the other hand, the other species and the root parts could not be stained. It was suggested that the established eastern blotting system based on anti-AA-I and AA-II MAb was considerably more specific than TLC method.

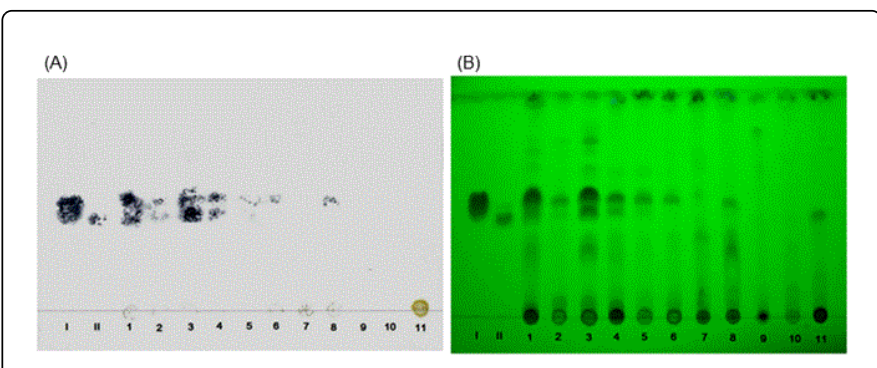

Figure 9: Fingerprinting of Aristolochea and Asarum species stem by eastern blotting (A) and UV on TLC (B).

\section{Licorice}

Glycyrrhiza species (Leguminosae) is perennial plants growing up to $1.5 \mathrm{~m}$ high. These plants are generally distributed in drylands between Western Europe to Russia and particularly abundant in China and Mongolia. Licorice, the root of Glycyrrhiza species, is listed in Material Medica and Shennong Ben Cao Jing as an important and safe medicine, and therefore it is well-known that one of the most important herb medicine used in TCM and prescribed with other herb medicine in $70 \%$ of prescription for a tussive, expectorant and corrigent, treatment of nervous, alimentary, respiratory, endocrine, and cardiovascular system diseases and so on [67]. These pharmaceutical properties are mainly due to GC which has protein inhibitory activity, anti-ulcer and anti-viral activities [68-70] and is now used for protection of the lever and as an anti-allergic during this fifty years in Japan [71]. Major species, G. uralensis Fisch (Figure 10) and G. glabra Linne are listed in the Japanese pharmacopoeia. Five hundred or more components have been identified in licorice, including GC which is controlled at a concentration of more than $2.5 \%$ and a flavonoid, Liq has a quantitative limitation in Japanese Pharmacopoeia too (Figure 11). Flavonoids have also wide pharmacologically active spectra like anti-hepatotoxic, antiinflammatory, anti-ulcer, anti-allergenic, and anti-viral activities as well as cardioprotective activities [72-76]. Licorice is also widely used as a sweetener in the production of confectioneries, soy sauce and is therefore in high demand worldwide. An anti-GC MAb was prepared in our laboratory [14] and we previously reported on the synergistic effects of GC and other constituents [37], the interfacial behavior of GC [77], and the identification of Glycyrrhiza species with higher GC content [27]. More recently we found that two flavonoids, isoliquiritigenin and formononetin promote in vitro fertilization [78].

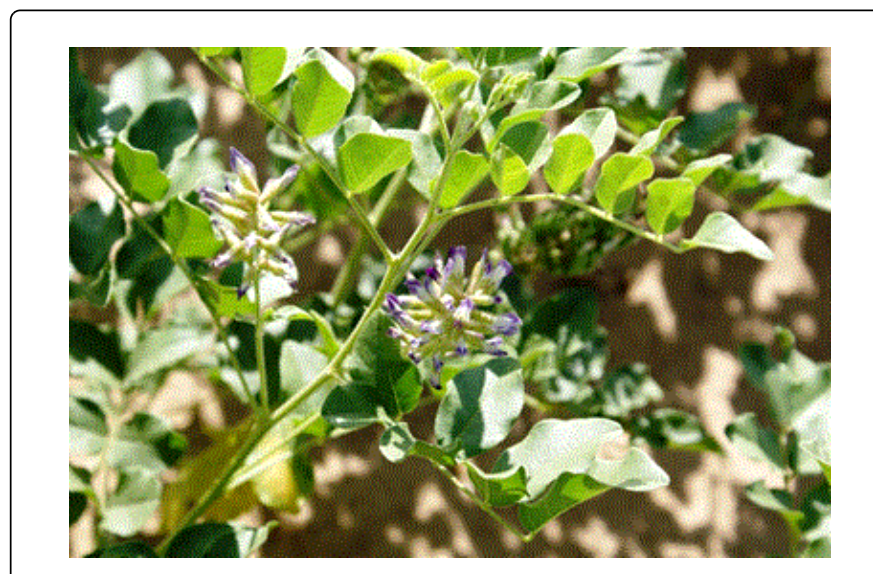

Figure 10: Glycyrrhiza uralensis Fisch and its flowering. 


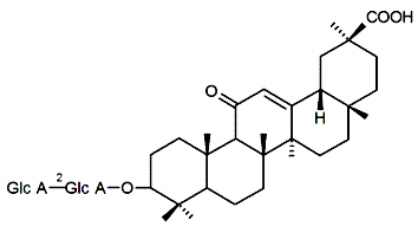

Glycyrrhizin
(Glycyrrhizic acid)<smiles>CC(C)=CCc1cc2c(=O)cc(-c3ccc(O)cc3)oc2cc1O</smiles>

Licoflavone<smiles>COc1cc2c(c(O)c1CC=C(C)C)CC(c1ccc(O)c(CC=C(C)C)c1O)CO2</smiles>

Licoricidin

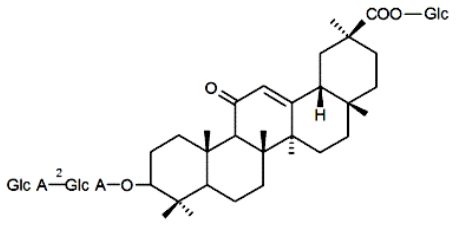

Licorice-saponin A3<smiles>COc1cc(O)c(-c2coc3cc(O)ccc3c2=O)c(OC)c1CC=C(C)C</smiles>

Licoricone

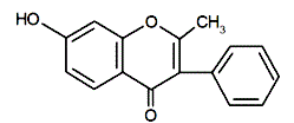

2-Methyl-7-hydroxyisoflavone<smiles>O=Cc1ccc2c(c1)OCc1ccc(O)cc1C2</smiles>

Liquiritin<smiles>COc1c(CC=C(C)C)c(O)cc2oc(=O)c3c4ccc(O)cc4oc3c12</smiles>

Glycyrol

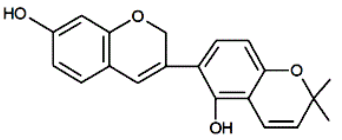

Glabrene<smiles>COc1ccc(/C=C/C(=O)c2ccc(O)cc2O)cc1</smiles>

Isoliquiritin<smiles>COc1c2c(cc3oc(=O)c4c5ccc(O)cc5oc4c13)OC(C)(C)CC2</smiles>

Isoglycyrol

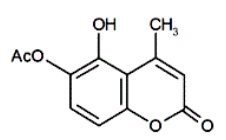

Liqcoumarin

Figure 11: Components in licorice.

GC and Liq are two major components in licorice that have wellknown pharmacological activities as documented above. Quality control of licorice root is needed to assure uniformity in the concentrations of these compounds, which can differ according to licorice species, growth plce, and harvest season, and so on. We also previously prepared MAbs against Liq [43] for quality control of licorice in order to make evident the resource of licorice for further researches.

We discussed eastern blotting for solasodine glycoside using antisolamargine MAb having wide cross-reactivity previously. On the other hand we prepared a high specific anti-GC MAb indicating that the cross-reactivity of aglycone of GC, glycyrrhetic acid and glycyrrhetic acid-mono-glucuronide were 2 and 4\%, respectively [14]. $\mathrm{MAb}$ against Liq is almost same with that of GC although an aglycone of Liq has lower cross-reactivity [14]. Therefore, double eastern blotting of GC and Liq was performed on the basis of the previously described methodology. Moreover, although a double eastern blotting had been developed for detecting structurally related compounds such as ginsenosides (dammarane-type triterpenoid saponin) [41] and sennosides (anthraquinone glycoside) [40], detecting two compounds that differ chemically such as GC (triterpenoid saponin) and Liq (flavonoid glycoside) has not been achieved yet.

GC, glycyrrhetinic acid, Liq, liquiritigenin and a sample extract solution were applied to the TLC plate and developed with butanolwater-acetic acid solvent system After blotting from the TLC plate to the PES membrane, the blotted membrane was treated with a sodium periodate solution and then treated with BSA solution. The membrane was treated with anti-GC MAb, peroxidase-labeled secondary antibody, and 3-amino-9-ethylcarbazole. For successive staining by anti-Liq MAb, the PES membrane was treated in the same manner as the anti-GC MAb except that it was exposed to 4-chloro-1-naphthol.
GC and Liq on the membrane can be detected by labeling with antiGC and anti-Liq MAbs (Figures 12B and 12C). However, two aglycones cannot be stained by eastern blotting (Figure 12B) because unlike TLC (Figure 12A), there are no sugar moieties that can be conjugated to the membrane as previously conformed in solasodine glycoside.

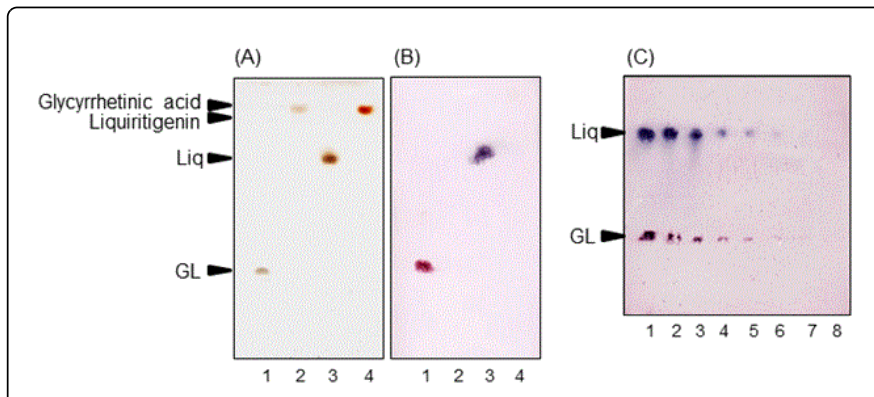

Figure 12: Double eastern blotting $(\mathrm{B}, \mathrm{C})$ using anti-GL and anti-Liq MAbs and sulfuric acid staining (A) for GL and Liq.

Samples $1 \sim 4$ are GC, glycyrrhethic acid, Liq and liquiritigenin in (A) and (B), respectively. Samples $1 \sim 8$ in (C) are GL and Liq standard solution as $20,10,5,2.5,1.25,0.63,0.31$ and $0.16 \mu \mathrm{g}$, respectively.

Because 4-chloro-1-naphthol and 3-amino-9-ethylcarbazole were compatible with a peroxidase-labeled secondary antibody, double eastern blotting was employed for these substrates. 4-Chloro-1naphthol was found to be unsuitable for GC analysis in licorice and licorice products because the bands were unclear. Instead, 3-amino-9ethylcarbazole was selected for GC staining, which give a reddish color. Thereby, Liq gave a purple color when stained with 4chloro-1- 
naphthol. These results demonstrate that 3-amino-9ethylcarbazole and 4-chloro-1-naphthol were suitable for the detection of GC and Liq, respectively. In evaluating the sensitivity of double eastern blotting, various concentrations of GC and Liq were detected using anti-GC and anti-Liq MAbs. The colored GC and Liq spots appeared by double eastern blotting at a minimum of $0.63 \mu \mathrm{g}$. Although GC, Liq, and their aglycones, glycyrrhetinic acid and liquiritigenin, can be detected by sulfuric acid staining, double eastern blotting using anti-GC and antiLiq MAbs can only detect GL and Liq. The advantage of using double eastern blotting compared to TLC analysis, is that it is possible to identify GC and Liq in impure or crude sample extracts without any standard compounds and their retention factors. Another advantage of this immunostaining system is that it enables identification of licorice from different plant species based on a one-step analysis, although licorice contains nearly 500 constituents, which normally would result in complicated component patterns, making it nearly impossible to identify licorice without suitable pretreatment using TLC and HPLC. Moreover, although several compounds and other crude drugs are present in Kampo medicine, the concentrations of GC and Liq in various Kampo medicines were in good agreement with the color depth and band size of the stains by double eastern blotting.

\section{Ginseng}

Genus Panax mainly includes 4 species, $P$ ginseng, $P$. japonicus, $P$. quinquefolium and $P$. notoginseng. Among them, $P$. ginseng was listed as the upper class medicine in Shén nóng běn căo jīng nearly 2 thousand years ago and has the centuries-old history. It has been used in forms of extract, powder, tea, tablet, capsule, etc., for their ability to enhance physical performance and for their adaptogenic effects, which are believed to increase the body's ability to fight stress, increase resistance to disease by strengthening normal body function, and reduce aging process. Phytochemical of ginseng has been studied extensively and, to date, more than 100 dammarane-type triterpene oligoglycosides, generally named as ginsenosides such as ginsenosides Rb1, Rb2, Rg1, Rd, Re, have been identified [79,80](Figure 13).

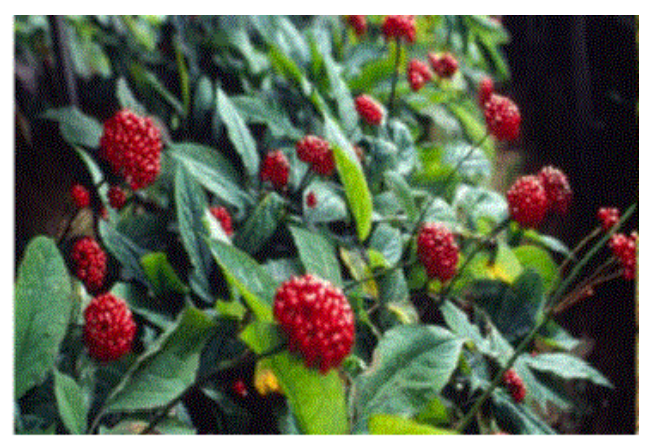

Figure 13: Panax ginseng C. A. Meyer and its fruiting.

Regarding eastern blotting we already discussed three types of compounds like solasodine glycosydes in Solanum spp., aristolochic acids in Aristolochia species and GL and Liq in Glycyrrhiza species. Solasodine glycosides are an example of determination for all solasodine glycosides using MAb having wide cross-reactivities. Next we discussed how to conjugate to membrane for compounds having no sugar moiety in a molecule using aristolochic acids. In licorice two types of compound like triterpene glycoside and flavonoid could be detected by double eastern blotting. In this section double eastern blotting system can be used for staining for ginseng saponins.

However, since 100 ginsenosides have been isolated and elucidated structures, the identification of individual ginsenosides is not easy. However, two MAbs against ginsenoside Rb1 and ginsenoside Rg1 can recognize almost all ginsenosides although two MAbs are specific $[81,82]$.

There are two types of ginsenosides related to aglycone, protopanaxdiol and protopanaxtriol. Therefore we prepared two MAb against ginsenoside Rb1 and $\mathrm{Rg} 1$ [6,7]. Figure 14 indicated two types of ginsenoside, ginsenoside $\mathrm{Rb} 1$ and ginsenoside $\mathrm{Rg} 1$. Difference between two ginsenosides is the position of glucose conjugation to aglycone. In the case of ginsenoside Rb1 no glucose conjugated at C6 positon. On the other hand ginsenoside $\operatorname{Rg} 1$ has one glucose conjugated at C6 hydroxyl group.
(A)

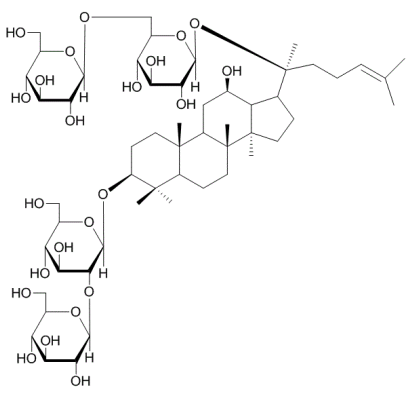

(B)

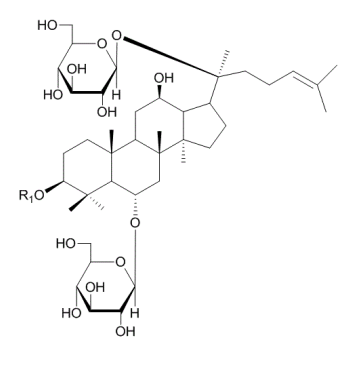

Figure 14: Structure of ginsenoside $\mathrm{Rb} 1$ (A) and ginsenoside $\mathrm{Rg} 1$ (B).

Figure 15 showed the double eastern blotting (B) and $\mathrm{H}_{2} \mathrm{SO}_{4}$ staining (A) of various Panax species. Pinkish spots indicated ginsenoside $\operatorname{Rg} 1$ and $\operatorname{Re}$ possessing protopanaxatriol as an aglycone. On the other hand, blue color showed ginsenoside Rb1 and Rc and so on which have protopanaxadiol in a molecule. It is suggested that the specific reactivity of the sugar moiety in the ginsenoside molecule against MAb may be fortunately modified by sodium periodide treatment of ginsenoside on the membrane resulting in wide crossreactivity although the protopanaxadiol and protopanaxatriol groups are still recognized each other. If anti-ginsenoside $\mathrm{Rb} 1 \mathrm{MAb}$ will be used, all ginsenosides having protopanaxadiol as an aglycone can be stained. Anti-ginsenoside Rg1 can stain almost all ginsenoside having protopanaxatriol as an aglycone. This double eastern blotting has the other function because ginsenosides of protopanaxadiol group have the suppressive activity for central nervous system [83]. On the other hand ginsenosides having protopanaxatriol indicate excite activity for central nervous system [84]. Therefore, Figure 15 indicates that blush color ginsenosides have the suppressive activity and pinkish ginsenosides have exciting activity. Therefore, it becomes evident that we will be able to evaluate the pharmacological activity against ginsenosides by double staining using anti-ginsenoside Rb1 and Rg1 MAb.

Since we have succeeded to determine the distribution of ginsenoside $\mathrm{Rb} 1$ in ginseng tissues [84,85], our previous result of pharmacokinetics for ginsenosides Rb1 and Rg1 [29] makes it possible clear the distribution of ginsenosides $\mathrm{Rb} 1$ and $\mathrm{Rg} 1$ in tissues. 
Furthermore, it might be possible to confirm the distribution of ginsenoside $\mathrm{Rb} 1$ and $\mathrm{Rg} 1$ in a brain by double eastern blotting using anti-ginsenosides Rb1 and Rg1 MAbs in order to make evident the relation between the structure of ginsenoside and pharmacological activity in central nervous system as suggested previously.

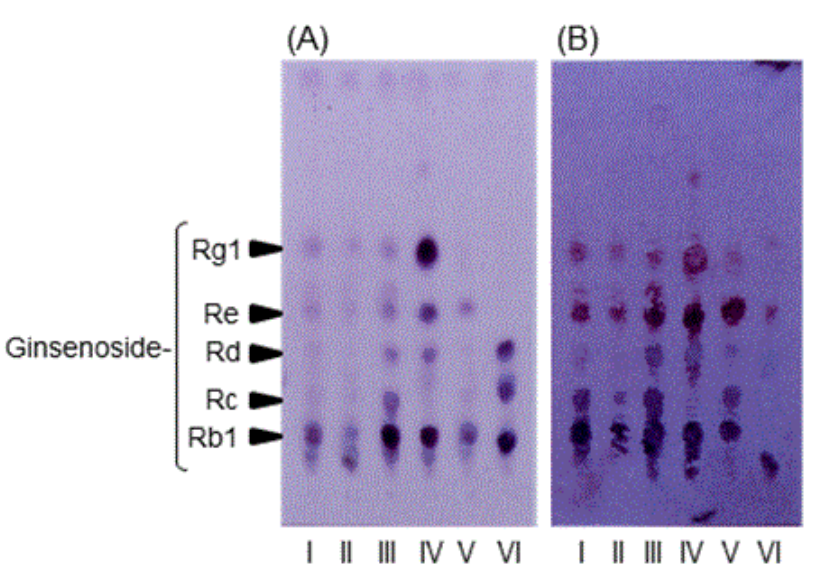

Figure 15: $\mathrm{H}_{2} \mathrm{SO}_{4}$ staining (A) and double eastern blotting (B) of various Panax spp. II, III, IV, V and VI indicated white ginseng, red ginseng, fibrous ginseng (Panax ginseng), Panax notoginseng, Panax quinquefolius and Panax japonicus, respectively. Upper purple color spots and lower blue color spots were stained by antiG-Rg1 and anti-G-Rb1 monoclonal antibodies, respectively.

\section{Conclusion}

In this review, we described the eastern blotting for quality control of natural products. Eastern blotting is an on-membrane immunestaining and quantitative analysis system for natural products. We introduced several examples such as solasodine glycosides, aristrochic acid, GC, Liq, and ginsenosides. The MAbs which we prepared have different cross-reactivities against each target compounds. By using the useful property of these MAbs, eastern blotting is specific and sensitive compared with TLC to determine the target compounds in crude extracts of medicinal plants. Double eastern blotting made possible to detect two different compounds in same membrane using different substrates for secondary antibodies. Moreover, the histochemical study of plant using eastern blotting is possible to investigate the distribution of target compounds in the sliced plant. In addition, eastern blotting also made possible the detection of target compounds in biological sample like tissue. These applications using eastern blotting may open the possibility to develop the quality control of natural products, and to apply the analysis of plant morphology and biological study related with natural products.

\section{References}

1. Sakata R, Shoyama Y, Murakami H (1994) Production of monoclonal antibodies and enzyme immunoassay for typical adenylate cyclase activator, forskolin. Cytotechnology 16: 101-108.

2. Ishiyama M, Shoyama Y, Murakami H, Shinohara H (1996) Production of monoclonal antibodies and development of an ELISA for solamargine. Cytotechnology 18: 153-158.
3. Xuan L, Tanaka H, Xu Y, Shoyama Y (1999) Preparation of monoclonal antibody against crocin and its characterization. Cytotechnology 29: 65-70.

4. Tanaka H, Goto Y, Shoyama Y (1996) Monoclonal antibody based enzyme immunoassay for marihuana (cannabinoid) compounds. J Immunoassay 17: 321-342.

5. Shoyama Y, Fukada T, Murakami H (1995) Production of monoclonal antibodies and ELISA for thebaine and codeine. Cytotechnology 19: 55-61.

6. Tanaka H, Fukuda N, Shoyama Y (1999) Formation of monoclonal antibody against a major ginseng component, ginsenoside Rb1 and its characterization. Cytotechnology 29: 115-120.

7. Fukuda N, Tanaka H, Shoyama Y (2000) Formation of monoclonal antibody against a major ginseng component, ginsenoside $\mathrm{Rg} 1$ and its characterization: Monoclonal antibody for a ginseng saponin. Cytotechnology 34: 197-204.

8. Kim JS, Tanaka H, Shoyama Y (2004) Immunoquantitative analysis for berberine and its related compounds using monoclonal antibodies in herbal medicines. Analyst 129: 87-91.

9. Morinaga O, Tanaka H, Shoyama Y (2000) Production of monoclonal antibody against a major purgative component, sennoside A, its characterization and ELISA. Analyst 125: 1109-1113.

10. Morinaga O, Nakajima S, Tanaka H, Shoyama Y (2000) Production of monoclonal antibodies against a major purgative component, sennoside $\mathrm{B}$, their characterization and use in ELISA. Analyst 126: 1372-1376.

11. Lu Z, Morinaga O, Tanaka H, Shoyama Y (2003) A quantitative ELISA using monoclonal antibody to survey paeoniflorin and albiflorin in crude drugs and traditional Chinese herbal medicines. Biol Pharm Bull 26: 862-866.

12. Morinaga O, Waraporn W, Sangmalee S, Lin L, Lu Z, et al. (2013) Detection of paeoniflorin and albiflorin by immunostaining technique using anti-paeoniflorin monoclonal antibody. Phytochem Anal 24: 124-128.

13. Tanaka H, Shoyama Y (1998) Formation of a monoclonal antibody against glycyrrhizin and development of an ELISA. Biol Pharm Bull 21: 1391-1393.

14. Shan S, Tanaka H, Shoyama Y (2001) Enzyme-linked immunosorbent assay for glycyrrhizin using anti-glycyrrhizin monoclonal antibody and an eastern blotting technique for glucuronides of glycyrrhetic acid. Anal Chem 73: 5784-5790.

15. Zhu S, Shimokawa S, Tanaka H, Shoyama Y (2004) Development of an assay system for saikosaponin a using antisaikosaponin a monoclonal antibodies. Biol Pharm Bull: 27: 66-71.

16. Morinaga O, Zhu S, Tanaka H, Shoyama Y (2006) Visual detection of saikosaponins by on-membrane immunoassay and estimation of traditional Chinese medicines containing Bupleuri radix. Biochem Biophys Res Commun 346: 687-692.

17. Chao Z, Cui Q, Tian E, Zeng W, Cai X, et al. (2016) Ultrasensitive timeresolved fluoroimmunoassay for saikosaponin a in Chaihu (Bupleuri Radix). PLoS One 11.

18. Loungratana P, Tanaka H, Shoyama Y (2004) Production of monoclonal antibody against ginkgolic acids in Ginkgo biloba Linn. Am J Chin Med 32: 33-48.

19. Kido K, Edakuni K, Morinaga O, Tanaka H, Shoyama Y (2008) An enzyme-linked immunosorbent assay for aconitine-type alkaloids using an anti-aconitine monoclonal antibody. Anal Chim Acta 616: 109-114.

20. Kido K, Morinaga O, Shoyama Y, Tanaka H (2008) Quick analysis of baicalin in Scutellariae Radix by enzyme-linked immunosorbent assay using a monoclonal antibody. Talanta 77: 346-350.

21. Yang Q, Zhu J, Ma F, Li P, Zhang W, et al. (2016) Quantitative determination of major capsaicinoids in serum by ELISA and timeresolved fluorescent immunoassay based on monoclonal antibodies. Biosens Bioelectron 81: 229-235.

22. Tanaka H, Putalun W, De-Eknamkul W, Matangkasombut O, Shoyama Y (2007) Preparation of a novel monoclonal antibody against the 
antimalarial drugs, artemisinin and artesunate. Planta Med 73: $1127-1132$.

23. Guo S, Cui Y, Wang K, Zhang W, Tan G, et al. (2016) Preparation of a novel monoclonal antibody against the antimalarial of artemisinin in Artemisia annua and rat serum. Anal Chem 88: 2701-2706.

24. Zhang B, Nan T, Zhan Z, Kang L, Yang J, et al. (2016) Development of a monoclonal antibody-based enzyme-linked immunosorbent assay for luteoloside detection in Flos Lonicerae Japonicae. Anal Bioanal Chem.

25. Yusakul G, Sakamoto S, Juengwatanatrakul T, Putalun W, Tanaka H, et al. (2016) Preparation and application of a monoclonal antibody against the isoflavone glycoside daidzin using a mannich reaction-derived hapten conjugate. Phytochem Anal 27: 81-88.

26. Qu H, Zhang Y, Qu B, Kong H, Qin G, et al. (2016) Rapid lateral-flow immunoassay for the quantum dot-based detection of puerarin. Biosens Bioelectron 81: 358-362

27. Fujii S, Tuvshintogtokh I, Mandakh B, Munkhjargal B, Uto T, et al. (2014) Screening of Glycyrrhiza uralensis Fisch. ex DC. containing high concentrations of glycyrrhizin by Eastern blotting and enzyme-linked immunosorbent assay using anti-glycyrrhizin monoclonal antibody for selective breeding of licorice. J Nat Med 68: 717-722.

28. Guo S, Cui Y, Wang K, Zhang W, Tan G, et al. (2016) Development of a specific monoclonal antibody for the quantification of artemisinin in Artemisia annua and rat serum. Anal Chem 88: 2701-2706.

29. Chao Z, Shoyama Y, Tanaka H (2006) Pharmacokinetic study of ginsenosides Rb1 and Rg1 in rat by ELISA using anti-ginsenosides Rb1 and Rg1 monoclonal antibodies. Am J Chin Med 34: 1069-1081.

30. Yanagihara H, Sakata R, Minami H, Tanaka H, Shoyama Y, et al. (1996) Immunoaffinity column chromatography against forskolin using an antiforskolin monoclonal antibody and its application. Anal Chim Acta 335: 63-70.

31. Fukuda N, Tanaka H, Shoyama Y (2000) Isolation of the pharmacologically active saponin ginsenoside $\mathrm{Rb} 1$ from Ginseng by immunoaffinity column chromatography. J Nat Prod 63: 283-285.

32. Putalum W, Tanaka H, Shoyama Y (1999) Rapid separation of solasodine glycoside by an immunoaffinity column using anti-solamargine monoclonal antibody. Cytotechnology 31: 151-156.

33. Xua J, Tanaka H, Shoyama Y (2007) One-step immunochromatographic separation and ELISA quantification of glycyrrhizin from traditional Chinese medicines. J Chromatogr B Analyt Technol Biomed Life Sci 850: 53-58.

34. Qu H, Zhang Y, Qu B, Cheng J, Liu S, et al. (2016) Novel immunoassay and rapid immunoaffinity chromatography method for the detection and selective extraction of naringin in Citrus aurantium. J Sep Sci 39: 1389-1398.

35. Uto T, Tuvshintogtokh I, Shoyama Y (2011) Preparation of knockout extract for determination of really active compound using MAb. Curr Drug Discov Technol 8: 16-23.

36. Shoyama Y (2011) Monoclonal antibodies against small molecule natural products and their applications, eastern blotting and knockout extract. Pharmaceuticals 4: 950-963.

37. Uto T, Morinaga O, Tanaka H, Shoyama Y (2012) Analysis of the synergistic effect of glycyrrhizin and other constituents in licorice extract on lipopolysaccharide-induced nitric oxide production using knock-out extract. Biochem Biophysic Res Commun 417: 473-478.

38. Tanaka H, Putalun W, Tsuzaki C, Shoyama Y (1997) A simple determination of steroidal alkaloid glycosides by thin-layer chromatography immunostaining using monoclonal antibody against solamargine. FEBS Lett 404: 279-282.

39. Zhu S, Shimokawa S, Shoyama Y, Tanaka H (2006) A novel analytical ELISA-based methodology for pharmacologically active saikosaponins. Fitoterapia 77: 100-108.

40. Morinaga O, Uto T, Sakamoto S, Putalun W, Tanaka H, et al. (2009) Development of eastern blotting technique for sennoside A and B using anti-sennoside A and B monoclonal antibodies. Phytochem Anal 20: 154-158.
41. Fukuda N, Tanaka H, Shoyama Y (1999) Western blotting for ginseng saponins, ginsenosides using anti-ginsenoside $\mathrm{Rb} 1$ monoclonal antibody. Biol Pharm Bull 22: 219-220.

42. Fukuda N, Tanaka H, Shoyama Y (2001) Double staining of ginsenosides by western blotting using anti-ginsenoside Rb1 and Rg1 monoclonal antibodies. Biol Pharm Bull 24: 1157-1160.

43. Fujii S, Morinaga O, Uto T, Nomura S, Shoyama Y (2014) Development of a monoclonal antibody-based immunochemical assay for liquiritin and its application to the quality control of licorice products. J Agric Food Chem 62: 3377-3383.

44. Mahao SB, Sahu NP, Ganguly AN, Kasai R, Tanaka O (1980) Steroidal alkaloids from Solanum khasianum: Applycation of 13C NMR spectroscopy to their structural elucidation. Phytochemistry 19: 2018-2020.

45. Debelle FD, Vanherweghem J, Nortier JL (2008) Aristolochic acid nephropathy: A worldwide problem. Kidney Int 74: 158-169.

46. Pepeljnjak S, Klaric MS (2010) Suspects in Etiology of Endemic Nephropathy: Aristolochic Acid versus Mycotoxins. Toxins 2: 1414-1427.

47. Kumar V, Poonam, Prasad AK, Parmar VS (2003) Naturally occurring aristolactams, aristolochic acids and dioxoaporphines and their biological activities. Nat Prod Rep 20: 565-583.

48. Arlt VM, Stiborova M, Schmeiser HH (2002) Aristolochic acid as a probable human cancer hazard in herbal remedies: a review. Mutagenesis $17: 265-277$.

49. Stiborova M, Frei E, Arlt VM, Schmeiser HH (2008) Metabolic activation of carcinogenic aristolochic acid, a risk factor for Balkan endemic nephropathy. Mutat Res 658: 55-67.

50. Shibutani S, Dong H, Suzuki N, Ueda S, Miller F, et al. (2007) Selective toxicity of aristolochic acids I and II. Drug Metab Dispos 35: 1217-1222.

51. Xing G, Qi X, Chen M, Wu Y, Yao J, et al. (2012) Comparison of the mutagenicity of aristolochic acid I and aristolochic acid II in the gpt delta transgenic mouse kidney. Mutat Res 743: 52-58.

52. Yeh Y, Lee Y, Hsieh H, Hwang D (2008) Short-term toxicity of aristolochic acid, aristolochic acid-I and aristolochic acid-II in rats. Food Chem Toxicol 46: 1157-1163.

53. Heinrich M, Chan J, Wanke S, Neinhuis C, Simmonds MSJ (2009) Local uses of Aristolochia species and content of nephrotoxic aristolochic acid 1 and 2-A global assessment based on bibliographic sources. J Ethnopharmacol 125: 108-144.

54. Vanherweghem JL, Depierreux M, Tielemans C, Abramowicz D, Dratwa M, et al. (1993) Rapidly progressive interstitial renal fibrosis in young women: association with slimming regimen including Chinese herbs. Lancet 341: 387-391.

55. Guo L, Yue H, Cai Z (2010) A novel pre-column fluorescent derivatization method for the sensitive determination of aristolochic acids in medicinal herbs by high-performance liquid chromatography with fluorescence detection. J Pharm Biomed Anal 53: 37-42.

56. Kuo C, Lee C, Lin S, Tsai IL, Lee S, et al. (2010) Rapid determination of aristolochic acids I and II in herbal products and biological samples by ultra-high-pressure liquid chromatography-tandem mass spectrometry. Talanta 80: 1672-1680.

57. Zhang CY, Wang X, Shang MY, Yu J, Xu YQ, et al. (2006) Simultaneous determination of five aristolochic acids and two aristololactams in Aristolochia plants by high-performance liquid chromatography. Biomed Chromatogr 20: 309-318.

58. Yu FY, Lin YH, Su CC (2006) A sensitive enzyme-linked immunosorbent assay for detecting carcinogenic aristolochic acid in herbal remedies. J Agric Food Chem 54: 2496-2501.

59. Nan TG, He SP, Tan GY, Li G, Wang BM, et al. (2010) Development of sensitive monoclonal antibody-based enzyme-linked immunosorbent assay for renal toxicity ingredient aristolochic acid $\mathrm{A}$ in Chinese herbal medicine. Chin J Anal Chem 38: 1206-1210.

60. Tian M, Tanaka H, Shang MY, Karashima S, Chao Z, et al. (2008) Production, characterization of a monoclonal antibody against 
Citation: Shunsuke Fujii, Nguyen Huu Tung, Takuhiro Uto, Hiroyuki Tanaka, Xiao-Wei Li, et al. (2016) Quality Control of Natural Products by Fingerprinting of Eastern Blotting. Pharm Anal Acta 7: 494. doi:10.4172/2153-2435.1000494

Page 11 of 11

aristolochic acid-II and development of its assay system. Am J Chin Med 36: $425-436$.

61. Li X, Morinaga O, Tian M, Uto T, Yu J, et al. (2013) Development of eastern blotting technique for the visual detection of aristolochic acids in Aristolochia and Asarum species using monoclonal antibody against aristolochic acids I and II. Phytochem Anal 24: 645-653.

62. Li X, Yokota S, Wang D, Wang X, Shoyama Y, et al. (2014) Localization of aristolochic acid in mouse kidney tissues by immunohistochemistry using an anti-AA-I and AA-II monoclonal antibody. Am J Chin Med 42: 1453-1469.

63. Wang D, Li X, Wang X, Tan H, Jia Y, et al. (2016) $\alpha$-actinin-4 is a possible target protein for aristolochic acid I in human kidney cell in vitro. Am J Chin Med 44: 291-304.

64. Shoyama Y, Sakata R, Isobe R, Murakami H, Kusai A, et al. (1993) Direct determination of forskolin-bovine serum albumin conjugate by matrxassisted laser desorption ionization mass spectrometry. Org Mass Spectr 28: 987-988.

65. Goto Y, Shima Y, Morimoto S, Shoyama Y, Murakami H, et al. (1994) Determination of tetrahydrocannabinolic acid-carrier protein conjugate by matrix-assisted laster desorption/Ionization mass spectrometry and antibody formation. Org Mass Spectr 29: 668-671.

66. Staros JV, Wright RW, Swingle DM (1986) Enhancement by Nhydroxysulfosuccinimide of water-soluble carbodiimide-mediated coupling reactions. Anal Biochem 156: 220-222.

67. Yang R, Wang LQ, Yuan BC, Liu Y (2015) The pharmacological activities of licorice. Planta Med 81: 1654-1669.

68. Aly Am, Al-Alousi L, Salem HA (2005) Licorice: a possible antiinflammatory and anti-ulcer drug. AAPS Pharm Sci Tech 6: E74-82.

69. Asi MN, Hosseinzadah H (2008) Review of pharmacological effects of Glycyrrhiza sp and its bioactive compounds. Phytother Res 22: 709-724.

70. Wang ZY, Nixon DW (2001) Licorice and cancer. Nutr Cancer 39: 1-11.

71. Hidaka I, Hino K, Korenaga M, Gondo T, Nishina S, et al. (2007) Stronger neo-minophagen $\mathrm{C}$, a glycyrrhizin-containing preparation, protects liver against carbon tetrachloride-induced oxidative stress in transgenic mice expressing the hepatitis $C$ virus polyprotein. Liver Int 27: 845-853.

72. Kim YM, Kim TH, Kim YW, Yang YM, Ryu DH, et al. (2010) Inhibition of liver $\mathrm{X}$ receptor- $\alpha$-dependent hepatic steatosis by isoliquiritigenin, a licorice antioxidant flavonoid, as mediated by JNK1 inhibition. Free Radic Biol Med 49: 1722-1734.

73. Xie YC, Dong XW, Wu XM, Yan XF, Xie QM (2009) Inhibitory effects of flavonoids extracted from licorice on lipopolysaccharide-induced acute pulmonary inflammation in mice. Int Immunopharmacol 9: 194-200.
74. Zhu L, Wei H, Wu Y, Yang S, Xiao L, et al. (2012) Licorice isoliquiritigenin suppresses RANKL-induced osteoclastogenesis in vitro and prevents inflammatory bone loss in vivo. Int J Biochem Cell Biol 44: 1139-1152.

75. Liu Y, Xie S, Wang Y, Luo K, Wang Y, et al. (2012) Liquiritigenin inhibits tumor growth and vascularization in a mouse model of HeLa cells. Molecules 17: 7206-7216.

76. Jayaprakasam B, Doddaga S, Wang R, Holmes D, Goldfarb J, et al. (2009) Licorice flavonoids inhibit eotaxin-1 secretion by human fetal lung fibroblasts in vitro. J Agric Food Chem 57: 820-825.

77. Sakamoto S, Shoyama Y, Uto T, Nakahara H, Shibata O (2013) Investigation of interfacial behavior of glycyrrhizin with a lipid raft model via a Langmuir monolayer study. Biochim Biophys Acta 1828: 1271-1283.

78. Tung NH, Shoyama Y, Wada M, Tanaka H (2015) Two activators of in vitro fertilization in mice from licorice. Biochem Biophys Res Commun 467: $447-450$.

79. Choi KT (2008) Botanical characteristics, pharmacological effects and medicinal components of Korean Panax ginseng C A Meyer. Acta Pharmacologica Sinca 29: 1109-1118.

80. Qi LW, Wang CZ, Yuan CS (2011) Isolation and analysis of ginseng: advances and challenges. Nat Prod Rep 28: 467-495.

81. Tanaka H, Fukuda N, Shoyama Y (2005) Isolation of sinsenoside Rb1 from Kalopanax pictus by Eastern blotting using anti-ginsenoside $\mathrm{Rb} 1$ monoclonal antibody. Phytother Res 19: 255-258.

82. Tanaka H, Fukuda N, Shoyama Y (2006) Identification and differentiation of Panax species using ELISA, RAPD and eastern blotting. Phytochem Anal 17: 46-55.

83. Kim HJ, Kim P, Shin CY (2013) A comprehensive review of the therapeutic and pharmacological effects of ginseng and ginsenosides in central nervous system. J Ginseng Res 37: 8-29.

84. Yokota S, Onohara Y, Shoyama Y (2011) Immunofluorescence and imunoelectron microscopic localization of medicinal substance, Rb1, in several plant parts of Panax ginseng. Curr Drug Discov Technol 8: 51-59.

85. Yokota S, Onohara Y, Uto T, Tanaka H, Morinaga O, et al. (2011) Localization of ginsenoside- $\mathrm{Rb} 1$ in Panax ginseng revealed by immunofluorescence and immunoelectron microscopic techniques. J Med Plants Res 5: 3176-3187. 